18

\title{
A computer model for tool management information system
}

\author{
R. V. Narang \\ Manufacturing Technology, Indiana-Purdue University, Fort Wayne, \\ USA \\ (219)481-6384,Fax:(219)481-5734 \\ e-mail: narang@cvax.ipfw.indiana.edu
}

\begin{abstract}
Tooling is an important and expensive part of any manufacturing enterprise. An application-based tool management system was developed for a machining company that had a total expenditure of over a million dollars per annum on tooling alone. The system was developed on a relational database system available on a PC network. All different types of tools, their cost, supplier location of the tooling, and other relevant information were built into the system for management to make the right decisions on tooling. The paper gives an overview of the tooling system and describes the individual modules and their interrelationships, including some of the important files. It also describes how the system helps the company controlling tool costs and inventory.
\end{abstract}

\section{Keywords}

Tooling management, automated manufacturing, data flow diagram, database, flexible manufacturing system, computer integrated manufacturing, management information system

\section{INTRODUCTION}

In a recent survey paper on tooling management, Gray et al. (1993) identified the lack of attention to tool management issues as the primary reason for the poor performance of 
many manufacturing facilities. The management of the increased number of tool components and their application requirements has also hindered increases in productivity.

Implementation of an encompassing tool management system is one of the most essential aspects of an effective automated manufacturing system (AMS). As automated manufacturing systems, especially flexible manufacturing systems (FMS), have become more common, there is evidence that a lack of attention to structured tool management has resulted in an increase in machine downtime, leading to a decrease in productivity. Many companies today cannot achieve on-time delivery or operating efficiency, not because of the lack of materials, machines or labor, but because of not having the right tooling at the right time.

In addition to being an integral part of an automated manufacturing system, tool management also has direct cost implications. Industry data show that tooling could account for $25 \%$ to $30 \%$ of production costs in an automated manufacturing system (Chen et al., 1993). Use of proper tool management methods in an AMS is thus critical in preventing losses due to operator error, overstocking and understocking of tooling, damaged tools, lost or misplaced tools, and poor quality parts. Indeed the growing recognition is that AMSs may operate with partial tool management, but the full potential of the new technology will not be realized without proper tool management.

The enthusiastic participation at the conferences on various issues of tool management demonstrates the pervasive interest among academia and practitioners. Because of the great interest directed toward tooling problems, papers addressing various issues of tool management have recently appeared in various journals including International Journal of Production Research, International Journal of Flexible Manufacturing Systems, Journal of manufacturing Systems, and Journal of Operations Management (Chen et al., 1993). A computerized tool management system should be used by companies aspiring to ISO 9000 . the Malcolm Baldridge Award or just cost cutting through better quality, organization and control. This paper describes a systematic way to design and develop a tool management relational model that can be adopted as a guide to develop tool management systems used in a computer integrated manufacturing (CIM) environment.

\section{TOOL DATABASE}

A tool database is the core of a tool management system in FMS. Tool management is not a single, simple activity, but it is composed of a complex and pervasive set of functions (Frechette, 1992). An important ingredient in management information systems is the database that represents the heart of the information system and provides a common data storage for consistency of application.

In particular a common database for all tools' data, which can be shared and retrieved by all work centers, will eliminate the redundancy and duplication of tool inventory, effort in tooling plans, and facilitate tool changeovers. For example, a production planner must know what tools can be used, how many useful tools exist and their whereabouts, otherwise a production plan cannot be scheduled properly. Production planning and process 
control systems must be updated or changed in real time, otherwise the operation of the system can be disrupted. Part programmers must know the actual sizes of the tools and their characteristics and behavior in different conditions, otherwise part programs cannot be generated properly, causing lower quality and productivity (Torvinen and Salminen, 1991).

A common database for all tooling and machining data provides the following advantages

First, it encourages tool standardization in the company, and this minimizes tool redundancy. Second, it can help to optimize tool selection. Third, it provides the user with a comprehensive pool of information with regard to cutting technology. It will aid in the selection of cutting tools, cutting fluid, speed, feed, depth of cut and tool change frequency for new operations. In addition, it improves tool inventory control and assists in rationalizing tool utilization. Furthermore, it increases the effectiveness of tool data monitoring and management systems. Finally, it provides valuable tool data for simulation studies (Elmaraghy, 1985). These benefits require a vast amount of analysis and system development work. Every piece of useful information must be identified so that the database can become the core of the tool management system in FMS.

Figure 1 illustrates the tool management system in a CIM environment. Note that from a tool management point of view, the links with the design and process planning must be emplasized. The links between tool management and other systems, such as accounting, purchases and inventory, are well known. In a complex CIM system, tooling information needs to be used at different times, for different purposes and by different users in a variety of subsystems, including production planning, process control, dynamic scheduling, part programming, tool preset and maintenance, assembly and stock control (Evershim, 1991).

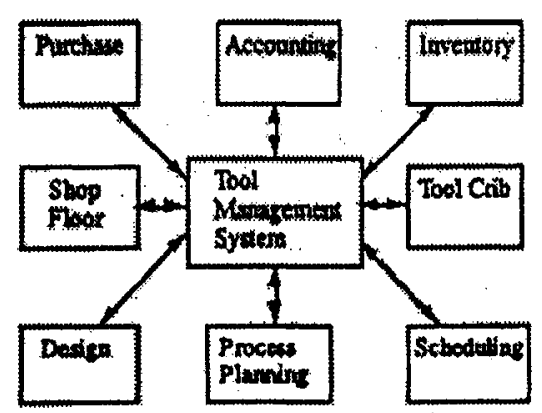

Bipure 1 Tool managernets system in CAM.

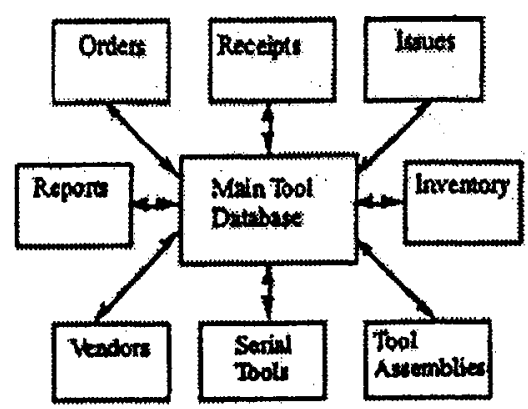

Figure 2 Modules in the tool management.

\section{TOOL MANAGEMENT MODEL}

The requirements of such a system were identified through integration of the collected information using tools such as DEF0 functional analysis and data flow diagranuming 
(DFD) technique. This became the basis for the design of relations and the database, which was implemented using a based relational database management system.

The IDEF0 methodology is a top-down hierarchical method providing a technique for modeling a complex manufacturing system. It can help to identify the information needs of the manufacturing system. From the integration of information point of view, constructing an IDEF0 model of a manufacturing system is the first step to understanding, documentation and implementation of a manufacturing system (Goodwin, 1989). Revision of IDEF0 models is recommended before any further developments. Figure 2 shows the role of the different tool databases in the tool management function. The following is a brief description of the major elements of the system.

Tools are received and stored initially as components. The main components for assembling complete tools are tool-holders and tool Inserts. According to the tool setup specifications, tool components are assembled, preset and, in some case, measured for offsets. The offsets data are used by the machining centers 10 detcrmine cutter compensation.

Groups of tools for specific operations are issued to the machine tool chain. Return of tools from machines can be due to wear-out, lack of immediate demand or lack of storage in the cell. Tools returning from the machines may be simply stored as assemblies, may be restored by regrinding or adjustment of inserts, or may be disassembled into components.

The geometric data of each tool-holder and tool insert are described by standard ISO codes (Shayan and Liu, 1995) which assist in designing an easy-to-use standardized database Figure 3 shows the parameters used to generate an ISO code for a turning tool-holder matching inserts for a $\mathrm{CNC}$ turning center. 


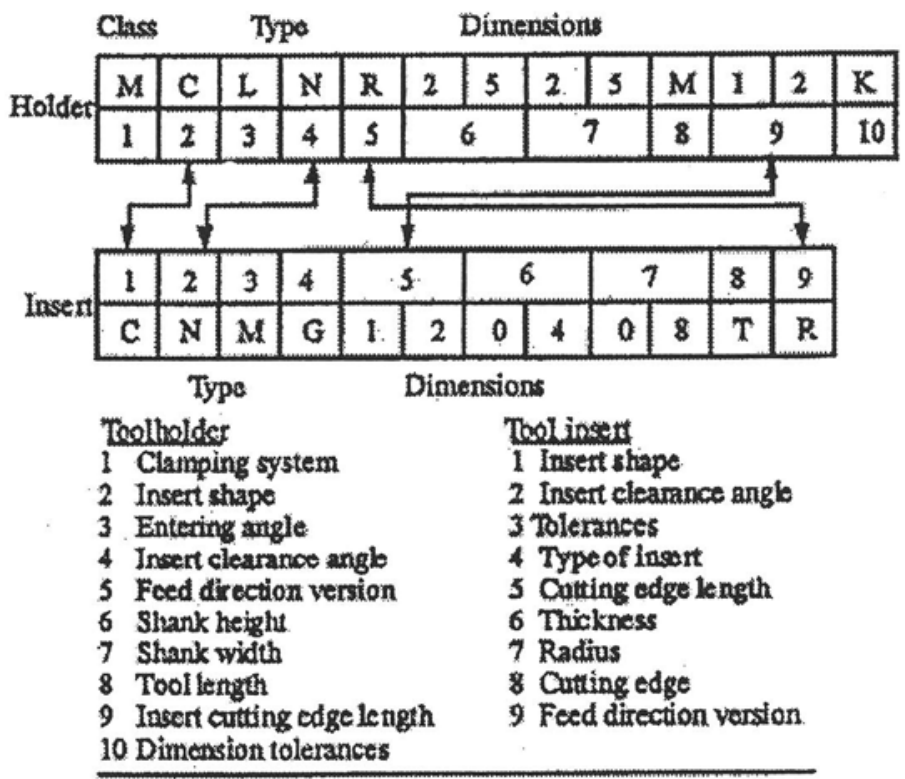

Figure 3 An ISO code for CNC turning center (Shayan and Liu, 1995).

A data flow diagram shows data moving about the system and being stored in data stores. DFDs yield a set, of data items of interest and their logical relationships. Figure 4 illustrates a data flow diagram for the tool receipts module. Every circle shows a process that may have a configuration of other activities.

The DFD diagramming technique is recognized as a good tool for logical database design by providing a natural and intuitive approach to model users' requirements, leading to system development. This forms the basis for further DFD analysis such as design of database files or tables and their fields. Some of the highlights of the tool management system are:

- Key fields in a table make that record unique in the entire table. For example, since serial number field is a key field in the Serial Tool table, different serial numbers will be required to be given to different tools.

- Stock category is the keyword by which a tool is classified such as, Hob, Drill, Insert, Shave Cutter, Blade, etc. The capital and small letters are critical for the system to identify the tools correctly. Table I shows some of tool category keywords. 


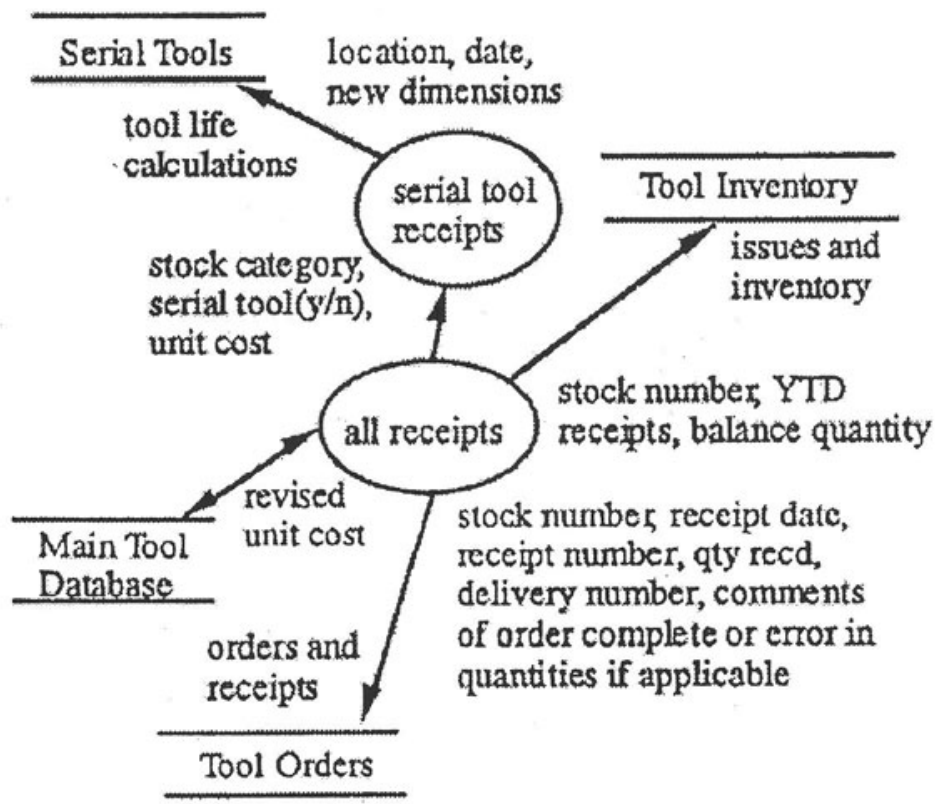

Figure $4 \mathrm{~A}$ data flow diagram for tool receipts module.

- The Main Tool database consists of a tool's unique record in the table. Certain repetitive type of information is asked once when it is encountered for the first time. That is, stock category, stock description, serial tool $(y / n)$, and vendor name are three specific items that are asked only once. Stock category, stock description, and serial tool are stored in Main Tool table. It also consists of important tool information, such as balance quantity, quantity on order, minimum quantity, system comments, total order costs, and total issue costs.

Table 1 Some examples of tool category

\begin{tabular}{|l|}
\hline TOOL CATEGORY \\
\hline Angular Milling Cutters \\
\hline Boring Bars \\
\hline Convex Cutters \\
\hline Corner Round Cutters \\
\hline Countersink Cutters \\
\hline Drills \\
\hline
\end{tabular}




\begin{tabular}{|l|}
\hline Endmills \\
\hline Indexable Inserts \\
\hline Milling Cutters \\
\hline
\end{tabular}

- The "System Comments" in the Main Tool table are given automatically by the system. The two types of comments are: (a) "Order Now" when the system detects the sum of balance quantity and quantity on order to be less than or equal to the minimum quantity, and (b) "On Order" when the system detects that the quantity on order is greater than 0 .

- Enter tool orders first and then their receipts. A purchase order may have multiple receipts. The system keeps track of the quantity received and closes the order automatically.

- If a particular information asked by the system is not available, the user can skip the question and can later update the table or form when the information becomes available.

- Tool issue summary by month is done in Tool Center table by the work center number and in Tool Category table by the tool category. Tool order summary by month is done in Tool Purchase table by the tool category. Year-to-date and the percentages are also calculated in these tables.

Tool management should provide information for decision making and problem resolution for:

- Manufacturing engineering: usage information will provide comparisons for new tool trials, check tool availability during fixture design, scrapped tool problem awareness.

- Industrial engineering: quickly locate tool numbers for process sheets, and utilize kits feature for process sheets.

- Accounting: tool expenditure accountability; inventory value.

- Purchasing: requisition review, vendor tracking, creation.

- Production Planning: check tooling availability for production schedule or "what if forecasting.

- Product engineering: reference in-house tool standards during design. 
- Production supervisors: eliminate time looking for tools, see problem areas by tool usage by work center.

- Tool-room: quick, easy tool ordering, no tool hunts, tool regrind or rework, know what work is in area by priority.

\section{IMPLEMENTATION}

Considering the properties of different database systems (network, hierarchical and relational) in terms of factors such as portability, ease of use, understanding and marketability, the choice was relational database (Elmasri, 1989). The choice of a PCbased database system was based on availability, size and reputation.

After the database was implemented, user interfaces to the database were developed. In order to design a user-friendly interface, development of forms-based applications for users to enter, query, update and print data is a desirable feature. Relational database management systems allow multiple views on the data. This property was exploited in generating different applications for a variety of potential users with the following requirements:

- Tool designers access the tool database to ensure that designs do not create new tool

- demands unnecessarily.

- Process planners access the database to ensure that only the available tools are specified

- for production

- Manufacturing engineering access the database to know where tools are used and monitor high volume users to ensure using the tools for the correct application and in an appropriate manner. They should be able to track the tool real-time data, such as remaining tool life and tool wear data from the tool database. They should also be able to qualify different grades for performance comparison, and costs can be more accurately assessed by

- product or work center.

- When tools are broken, the alternative tools should be identifiable in a very timely fashion.

- Part programmers access the database to find out the cutting parameters for specific tool inserts corresponding to specific work material.

The user selects the appropriate option from the top level menu to initialize the relevant module. The user can then query the database by the subsequent dialogue. It offers extensive tool life auditing capabilities based on tool wear. The major benefits are tracking of standard and serialized tooling through the regrind, and possibly, the inspection and coating areas, and for certain types of serialized tools, recording and calculating usage, wear, and remaining production capacity.

Standard perishable tooling such as drills can be returned from the shop floor directly into 
regrind area. After the tools are reworked, they are transferred to tool-room stock. Reports show where tools are and what was reworked, how many, by which employees, and over what time period.

For serialized tooling such as hobs and punch sets, re-sharpening is the only way to regain use of the tool after it has been dulled. The regrinding process removes valuable cutting metal until repeated regrinds result in a scrapped tool. Knowing the current production capacity of a tool in parts before it needs regrinding helps in determining if more tools should be purchased without overbuying. Additionally, knowing the total production capacity in part quantity of all tools enables the production planner to schedule jobs. Information collected for serialized tooling is minimum, maximum, and current dimension, regrind date, parts produced, theoretical and actual grinds, in order to form a life history of each tool. On-screen and printed reports provide valuable data on:

- current condition and used/remaining capacity per tool;

- complete tool wear histories

- machine performance by part number and operation;

- overall production capacity per tool group.

\section{CONCLUSION}

The requirements of a tool management system were identified through integration of the collected information. The methodology used to develop the system was IDEF0 functional analysis technique and DFD diagramming. The system was developed and implemented according to the framework presented herein. By making such a tool database available in day-to-day operation, savings in tool costs have been considerable. It also provides ease of tool management in satisfying requests for tools required for production. The tool database is a real and essential ingredient in the integration for the AMS environment. The system developed provides a basis for further specific developments.

\section{REFERENCES}

Chen, I. J., Gupta, A. and Chung, C-H.(1993) Tool planning and control: A selected bibliograply. Production and Inventory Management Journal, Third Quarter, 87-8.

Elmaraghy, H.A. (1985) Automated tools management in flexible manufacturing. Journal of Manufacturing Systems, 4, 1-13.

Elmasri, R. (1989) Fundamentals of Database Systems. Benjamin Cumminors, Redwood City, CA. 
Evershim, W. (1991) Tool management: the present and the future. Annals of the CIRP, 40,631-39.

Frechette, S. (1992) Open architecture for tool management. Manufacturing International, 319-34.

Goodwin, A.N., et al. (1989) An assessment of the IDEF notations as descriptive tools.

Information Systems, 14, 13-28.

Gray, A.E., Seldmann, A. and Stecke, K.E. (1993) A synthesis of decision models for tool management in automated manufacturing. Management Science, 39, 549-67.

Shayan, E. and Liu, C-L. (1995) Tool management in flexible manufacturing systems. Integrated Manufacturing Systems, 6, 26-3 5 .

Torvinen, S.J. and Salminen, K. (1991) Integration of a CIM tool management system to an intelligent feature-based process planning system. Computers in Industry, 17, 207-16.

\section{BIOGRAPHY}

Prof. R V. Narang is an associate professor at Indiana-Purdue University in the Manufacturing Technology department. He did his M.S. and Ph.D. in Industrial Engineering from the University of Iowa in 1975 and 1992 respectively. He has many years of industrial, teaching, and consulting experience. He is an ABET/TAC program evaluator for manufacturing engineering technology. He has presented a number of research papers in international conferences and published papers in reputed journals. 